\title{
Single-shot detection of wavepacket evolution
}

\author{
M.B. Campbell, T.J. Bensky, and R.R. Jones \\ University of Virginia, Charlottesville, VA, USA
}

\begin{abstract}
We have developed a new instrument for monitoring electronic wavepacket dynamics using a single electromagnetic pulse pair. The operation of the device is analogous to that of single-shot crosscorrelators commonly used to monitor the temporal evolution of short laser pulses. We have used the instrument to probe wavepacket evolution over time scales ranging from 100 psec to less than $1 \mathrm{fsec}$. The device reduces the amount of time required to collect pump-probe time delay data by orders of magnitude, greatly reducing the deleterious effects of experimental drifts. In addition, the single-shot feature provides real-time feedback as to the affect of various experimental parameters on the electron dynamics, allowing us to literally tune-up our equipment to enhance desired behavior at specific times.
\end{abstract}

\section{References}

1. R.R. Jones and L.D. Noordam, "Electronic Wavepackets," in Adv. At. Mol. Opt. Phys., Vol. 38 (in press).

2. L.D. Noordam and R.R. Jones, "Probing Rydberg Electron Dynamics," J. Mod. Optics (in press).

3. N.F. Scherer, A.J. Ruggiero, M. Du, and G.R. Fleming, "Time resolved dynamics of isolated molecular systems studied with phase-locked femtosecond pulse pairs," J. Chem. Phys. 93, 856 (1990).

4. N.F. Scherer, R.J. Carlson, R.J. Matro, M. Du, A.J. Ruggiero, V. Romero-Rochin, J.A. Cina, G.R. Fleming, and S.A. Rice, "Fluoresence-detected wave packet interferometry: Time resolved molecular spectroscopy with sequences of femtosecond phase-locked pulses," J. Chem. Phys. 95, 1487 (1991).

5. L.D. Noordam, D.I. Duncan, and T.F. Gallagher, "Ramsey fringes in atomic Rydberg wave packets," Phys. Rev. A 45, 4734 (1992).

6. B. Broers, J.F. Christian, J.H. Hoogenraad, W.J. van der Zande, H.B. van Linden van den Heuvell, and L.D. Noordam, "Time-resolved dynamics of electronic wavepackets above the classical field-ionization threshold," Phys. Rev. Lett. 71, 344 (1993).

7. R.R. Jones, D.W. Schumacher, T.F. Gallager, and P.H. Bucksbaum, "Bound-state interferometry using incoherent light," J. Phys. B 28, 405 (1995).

8. R.R. Jones, "Creating and Probing Electronic Wave Packets Using Half-Cycle Pulses," Phys. Rev. Lett. 76, 3927-3930 (1996).

9. D. You, R.R. Jones, and P.H. Bucksbaum, "Generation of high-power sub-single-cycle 500 fs electromagnetic pulses," Opt. Lett. 18, 290 (1993).

10. R.R. Jones, D. You, and P.H. Bucksbaum, "Ionization of Rydberg atoms by subpicosecond halfcycle electromagnetic pulses," Phys. Rev. Lett. 70, 1236 (1993).

11. C. Raman, C.W.S. Conover, C.I. Sukenik, and P.H. Bucksbaum, "Ionization of Rydberg Wave Packets by Subpicosecond, Half-Cycle Electromagnetic Pulses," Phys. Rev. Lett. 76, 2436-2439 (1996). 
During the last decade a large number of experiments have focused on the study of a variety of different types of electronic wavepackets in atoms.[1,2] Almost invariably, these experiments employ "pump" and "probe" laser pulses to create and monitor wavepacket evolution. The pump pulse populates a coherent superposition of electronic levels from some initial state. The evolution of this non-stationary state is reflected in the time-dependence of its stimulated photoabsorption and emission cross sections. Therefore, the motion of the wavepacket can be monitored experimentally using the probe pulse to (de)excite the wavepacket to some final state. Depending on the specifics of the experiment, different types of information on the electron dynamics can be obtained by measuring the electronic population in the final state as a function of the relative delay between the pump and probe pulse.[1,2]

Unfortunately, this type of measurement is destructive. The wavepacket that is produced by the pump pulse is destroyed by the probe. Therefore, the time-dependent evolution of the wavepacket can only be obtained from multiple measurements at different relative delays between the pump and probe. All experimental parameters must remain perfectly constant during consecutive measurements as the relative pump-probe delay is varied if an accurate determination of the electron dynamics is to be made. Of course, rapid shot-to-shot variations can be eliminated by averaging the results of multiple measurements at the same pump-probe delay. However, this necessarily increases the amount of time required to obtain a full delay scan and increases the possibilities for long term drifts, particularly in low repetition rate systems. In some situations, maintaining the required stability during hours of data collection makes experiments prohibitively difficult. Moreover, the destructive experimental method lacks real-time feedback. A delay scan must be performed before one can ascertain the affect on the dynamics of changing any experimental parameter.

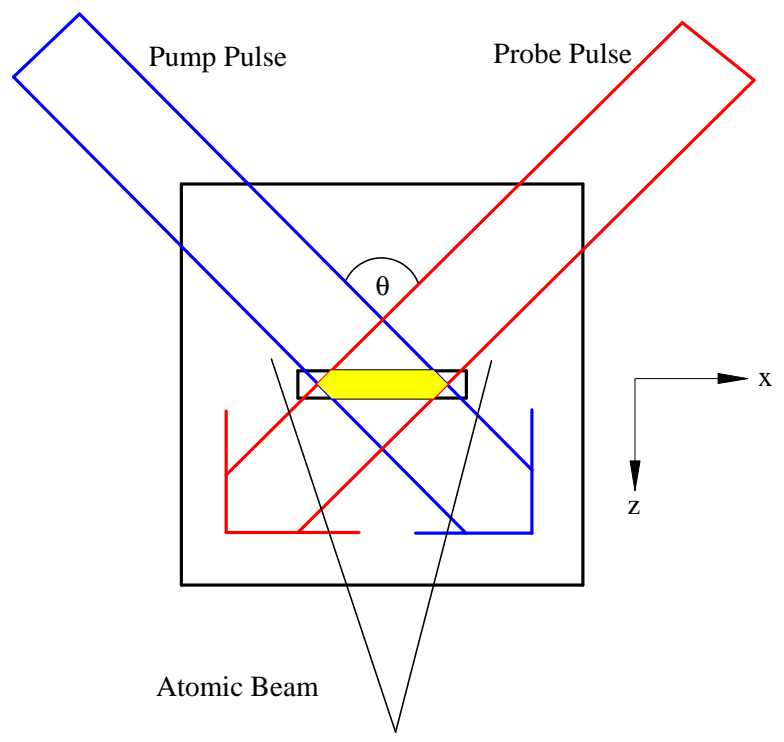

Figure 1. Operation schematic for a single-shot detector. 
In an attempt to circumvent these experimental problems, we have constructed a device which is capable of monitoring electron dynamics using a single pump-probe pair. The detector utilizes pump and probe beams which cross at some non-zero angle, $\theta$, through a sample of atoms as shown in Fig. 1. The relative pump-probe delay varies linearly along an axis, $\hat{x}$, perpendicular to the average propagation direction of the two pulses, $\hat{z}$. The difference in the relative pulse delay for two atoms separated by a distance, $\mathrm{d}$, along the $\hat{x}$ axis is $\Delta \tau=\frac{2 d}{c} \sin (\theta / 2)$. Therefore, in a single shot, atoms which experience a range of delays are present in the sample, and the problem of determining the final state population as a function of time-delay becomes one of measuring the number of final state atoms as a function of position along the $\hat{x}$ axis. This measurement is straightforward if the final state is in the continuum or can be selectively coupled to the continuum through subsequent photo-, field-, or auto-ionization.

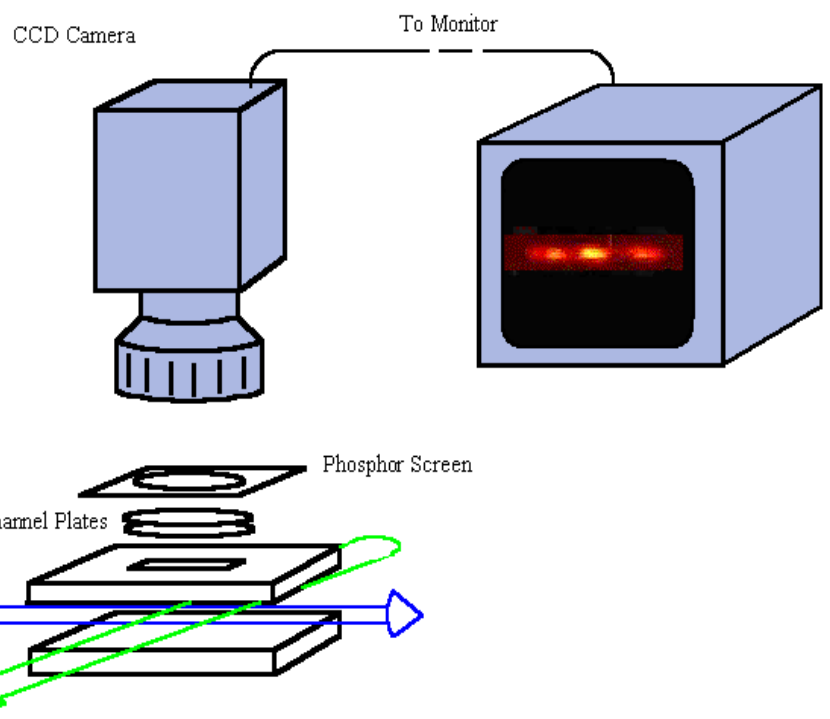

Figure 2. Schematic of the single-shot detector.

We have built an imaging detector capable of recording the spatially dependent ion signal produced by a single pump-probe pair in a crossed beam geometry. A schematic diagram of the detector is shown in Figure 2. A thermal beam of atoms propagates between two field plates that are separated by $1.5 \mathrm{~cm}$. A long slit $(2.5 \mathrm{~cm}$ $\mathrm{x} 0.16 \mathrm{~cm})$ in the upper field plate is oriented with its long dimension along the $\hat{x}$ axis. A voltage applied to the lower field plate pushes any ions in the interaction region through the slit in the upper plate. The details of the experiment dictate how the ions are actually produced, either directly by the probe pulse or through subsequent photo, auto-, or field-ionization of a final state populated by the probe. In any case, ions travel out of the interaction region and strike a microchannel plate detector with their relative positions preserved. Amplified electron current from the microchannel plates is accelerated towards a phosphor screen (Kimball Physics, ZnS:Ag Type 1330), and the fluoresence from the phosphor is imaged with a CCD camera. The ion distribution in the interaction region is reflected in the brightness of the CCD output as viewed on a television monitor. The CCD output is also transferred to a personal computer using a digital oscilloscope. 
The relative angle, $\theta$, between the propagation directions of the pump and probe beams and the physical size of the phospor screen determine the maximum single-shot delay that can be obtained. For counter propagating beams, we can observe time delays of approximately $100 \mathrm{psec}$ with the current apparatus. The spatial resolution of the imaging detector determines the temporal resolution of the measurement for a given beam geometry. With the current detector, the smallest feature that can be observed is approximately $300 \mu \mathrm{m}$, limited primarily by bleeding of the flouresence in the phosphor. Therefore, in the counter propagating beam geometry the resolution is approximately 1 psec. However, for nearly co-linear beams, the maximum time sweep across the detector can be made arbitrarily small. In fact, we have obtained sub-femtosecond resolution in this geometry. It is important to note that the detector can be used in conjunction with mechanical translation stages to extend the maximum achievable delay range. Singleshot data can be taken at different "macro-step" pump-probe delays by increasing the optical path length of the probe beam.

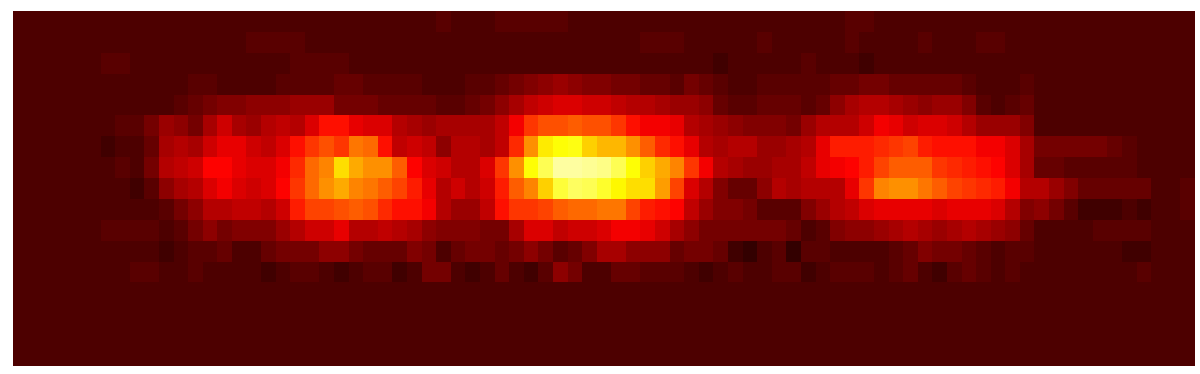

Figure 3. CCD image of the spatial distribution of the field ionization signal from a $\mathrm{Ry}-$ dberg wavepacket probed using bound-state interferometry in the single-shot configuration. The ion distribution reflects the temporal wavepacket interference. The period of the observed fringes is 1.3 fsec.

The imaging detector can be used in a wide variety of pump-probe experiments. Two applications which demonstrate the high and low resolution extremes are presented here. First, we have used the imaging detector to resolve the fast phase evolution of a Rydberg wavepacket probed using bound-state interferometry.[1-7] Ground state Ca atoms are excited to the $4 \mathrm{~s} 4 \mathrm{p}$ level using a nsec dye laser. A $500 \mathrm{fsec}, 392 \mathrm{~nm}$ laser pulse then drives the $4 \mathrm{~s} 4 \mathrm{p}$ - 4snd transition producing a Rydberg wavepacket centered near $\mathrm{n}$ $=25$. A second, identical $392 \mathrm{~nm}$ pulse enters the laser/atom interaction region nearly co-linearly with the first psec pulse. The two identical pulses propagate along the $\hat{z}$ axis, nearly perpendicularly to the slit in the upper field plate. The delayed pulse excites a second wavepacket that interferes with the original wavepacket. Approximately $1 \mu \mathrm{sec}$ after the laser excitation, a voltage pulse applied to the lower field plate ionizes the Rydberg atoms and pushes the resulting ions toward the imaging detector. Figure 3 shows the interference pattern produced by a single pump-probe pair as viewed with the CCD camera. The relative delay between the pump and probe beams is significantly longer than their pulse duration so there is no intensity variation due to optical interference. Instead, the sinusoidal intensity variation is strictly an atomic response to the two delayed pulses. The total number of Rydberg atoms produced by the pulse pair oscillates as the phase difference between the two wavepackets changes with varying pulse delay. The relative delay increase across each fringe is only 1.3 fsec.

Single-shot scans such as the one shown in Fig. 3 can be used to produce an autocorrelation of the wavepacket without relying on RMS signal averaging. [6,7] The signal level or power at the optical transition frequency can be obtained by taking the discrete Fourier transform of the single-shot phase delay scans. Therefore, one can mea- 
sure the interference level (i.e. the wavepacket autocorrelation function) as a function of time by macro-stepping the optical path length between the pump and probe pulses using a translation stage. The interference signal is obtained at each macro-step from the Fourier transform of the single-shot interference pattern. This method is quite fast. Furthermore, the signal-to-noise ratio of the resulting interferogram can be quite good since the Fourier transform rejects signal variations not at the optical frequency.

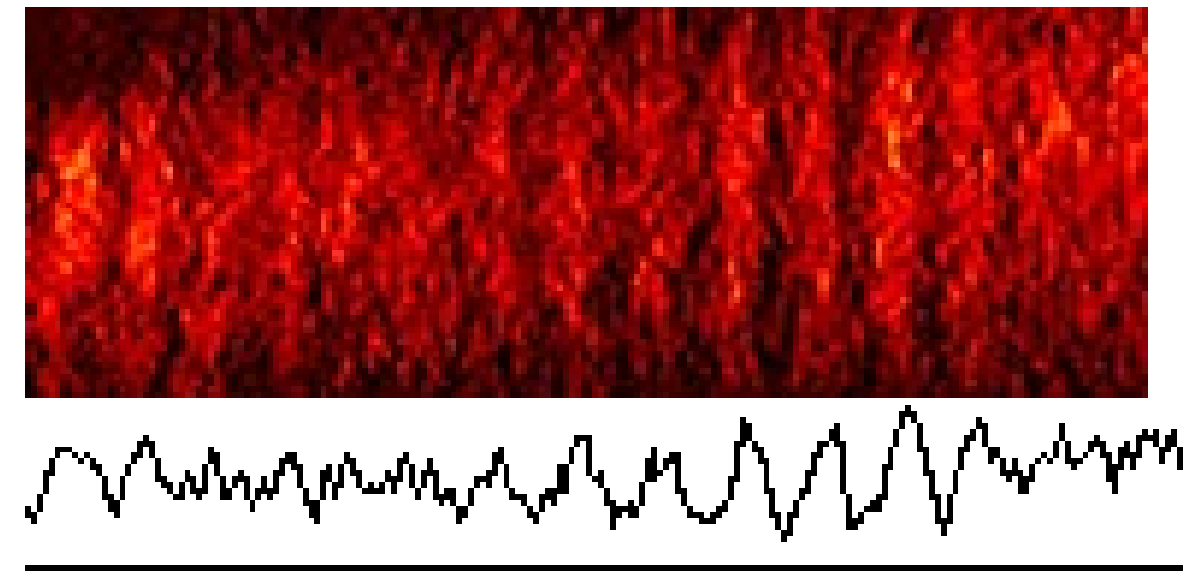

Figure 4. Single-shot image of wavepacket evolution obtained using a HCP probe in the counter-propagating beam geometry. The pump-probe delay increases from left to right and the interval across the full image is approximately 60 psec. The lower curve shows the intensity level integrated over the vertical axis in the image. Zero delay is near the left edge of the Figure.

A second application of the imaging detector is the direct observation of wave packet momentum evolution using impulsive momentum retrieval.[8] As in the previously described experiment, Ca atoms are excited to the $4 \mathrm{~s} 4 \mathrm{p}$ intermediate state to facilitate the creation of a 4 snd wavepacket using a $500 \mathrm{fsec}$ pulse of $392 \mathrm{~nm}$ radiation. The wavepacket is centered near $\mathrm{n}=30$ and consists of approximately 5 states. The two excitation pulses propagate along the $\hat{x}$ axis, directly beneath the slit in the upper field plate. The probe, a 500 fsec "half-cycle" pulse ( $\mathrm{HCP})$ of $\mathrm{THz}$ radiation $[9]$ propagates through the laser/atom interaction region anti-parallel to the pump pulse. The HCP gives the electronic wavepacket in each atom an impulsive "kick." [10] The energy distribution of the wavepacket following the kick depends on the size of the kick and the momentum distribution of the wavepacket before the kick.[10,11] By monitoring the ionization probability as a function of time and HCP field strength, the time-dependent momentum distribution of the wavepacket can be obtained.[8] Figure 4 shows the spatially dependent ionization signal produced by a single pump/HCP probe combination. The clear modulations in the ionization probability are due to the temporal evolution of the momentum distribution in the radial wavepacket. From left to right, the first few oscillations of the wavepacket followed by its collapse and full revival can be seen clearly in real time. Of course in an actual experiment, the signal to noise in the image can be improved dramatically by averaging the results from several laser shots. Even if two hundred shots are averaged, recording $100 \mathrm{psec}$ of time evolution at 50 different $\mathrm{HCP}$ field values requires less than 15 minutes with a $15 \mathrm{~Hz}$ laser repetition rate. Previous measurements using a convential spatially integrating detector required approximately $1 / 2$ hour for a single 100 psec time scan. [8] In that case, an entire day of data collection without any equipment drift is required to obtain a single momentum distribution. With 
the current apparatus several distributions can be collected in less than one hour with better statistics.

One of the most exciting features of the imaging detector is that it gives us the ability to tune up an experiment in real time. We essentially have an oscilloscope which immediately displays information on the phenomenon of interest. This allows us to optimize and maintain experimental parameters quickly and efficiently. The time needed to explore the dynamical effect of some experimental parameter is now several minutes, as opposed to hours, eliminating experimental instabilities such as laser drift or atomic source fluctuations. Experiments once considered too time consuming are now readily performed using the imaging detector.

We note that only one dimension of the detection slit is currently utilized. One can also consider using the narrow dimension of the slit to explore a second degree of freedom with each pump-probe pair. By spatially chirping the frequency spectrum of the pump and/or probe pulse, time vs. frequency information can be obtained in a single shot. If static electric or magnetic fields are used, a field gradient could be placed across the slit so that temporal dynamics vs. field strength could be monitored in a single shot.

In summary, we have demonstrated the use of a detector that is capable of monitoring wavepacket evolution with a single electromagnetic pump-probe pulse pair. This device facilitates data acquisition and allows for real-time tuning of an experiment to produce particular electronic configurations at specific times.

It is a pleasure to acknowledge the support of the AFOSR, the ONR, and the Packard Foundation. We would also like to thank B.L. Wood for technical assistance during the early stages of this work. 